\title{
MULLER'S RATCHET AND MUTATIONAL MELTDOWNS
}

\author{
W. GABRIEL, ${ }^{1}$ M. LYNCH, ${ }^{2}$ AND R. BÜRGER ${ }^{3}$ \\ ${ }^{1}$ Department of Physiological Ecology, Max Planck Institute for Limnology, \\ Postfach 165, D-24302 Plön, Germany \\ ${ }^{2}$ Department of Biology, University of Oregon, Eugene, Oregon 97403 \\ ${ }^{3}$ Institut für Mathematik der Universität Wien, \\ Strudlhofgasse 4, A-1090 Wien, Austria
}

\begin{abstract}
We extend our earlier work on the role of deleterious mutations in the extinction of obligately asexual populations. First, we develop analytical models for mutation accumulation that obviate the need for time-consuming computer simulations in certain ranges of the parameter space. When the number of mutations entering the population each generation is fairly high, the number of mutations per individual and the mean time to extinction can be predicted using classical approaches in quantitative genetics. However, when the mutation rate is very low, a fixationprobability approach is quite effective. Second, we show that an intermediate selection coefficient $(s)$ minimizes the time to extinction. The critical value of $s$ can be quite low, and we discuss the evolutionary implications of this, showing that increased sensitivity to mutation and loss of capacity for DNA repair can be selectively advantageous in asexual organisms. Finally, we consider the consequences of the mutational meltdown for the extinction of mitochondrial lineages in sexual species.
\end{abstract}

Key words. - Extinction, Muller's ratchet, mutations, selection coefficient.

Received June 22, 1992. Accepted April 6, 1993.

Deleterious mutations appear to arise at a rather high rate in most organisms, perhaps as high as one per gamete (Crow and Simmons 1983; Bell 1988a; Kondrashov 1988; Charlesworth et al. 1990; Houle et al. 1992). Much effort has been expended to determine whether populations can withstand this constant onslaught of mutations without suffering a substantial loss in individual fitness (Haldane 1937; Kimura et al. 1963; Crow and Kimura 1979; Kondrashov 1988; Charlesworth 1990). All other things being equal, populations that carry a higher mutation load should also have a higher risk of extinction.

Muller (1964) pointed out the special severity of this problem for small asexual populations. Assuming back mutations are rare, in the absence of recombination, no individual can ever produce an offspring with fewer deleterious mutations than it carries itself. The possibility always exists that, by chance, the class of individuals with lowest fitness will not produce offspring in some generation. After this class of individuals has been lost, the second-best class is expected to ultimately suffer the same fate, and so on. Felsenstein (1974) called this phenomenon Muller's ratchet, and he and others (Haigh 1978; Maynard Smith 1978; Pamilo et al. 1987; Bell 1988a,b; Birky and Walsh 1988; Charlesworth 1990; Lynch and Gabriel 1990; Melzer and Koeslag 1991) have performed theoretical work on the rate of accumulation of deleterious mutations in finite asexual populations. However, with the exception of Bell (1988a,b), Lynch and Gabriel (1990), and Melzer and Koeslag (1991), all of this work has assumed that population size is unaffected by the number of accumulated mutations. Such an assumption seems rather inconsistent with the notion that the mutations are harmful. In effect, it confines attention to the relative fitness of individuals within a population, with no consideration being given to the consequences of the absolute loss of fitness for population survival.

Recently, we investigated the connection between the operation of Muller's ratchet and the expected longevity of asexual populations (Lynch and Gabriel 1990), focusing especially on the positive feedback that arises between mutation accumulation and random genetic drift. By reducing the mean absolute fitness of individuals, mutation accumulation is expected to result eventually in a decline in population size. But in a smaller population, chance plays a greater role, thus subsequent deleterious mutations accumulate with a slightly higher probability, causing a somewhat more rapid decline in mean fitness and population size. Thus, the decay in fitness is expected to occur at an accelerating rate, a phenomenon that we refer to as a mutational meltdown. 
Previous work on Muller's ratchet, under the restrictive conditions mentioned above, led to the conclusion that the rate of mutation accumulation decreases as the effect of the mutation increases. However, using computer simulation, we showed that this can be offset by the reduction in fitness per fixed number of mutations, such that the expected time to extinction declines with increasing selection coefficient (Lynch and $\mathrm{Ga}-$ briel 1990). The more extensive analyses presented below show that this is not strictly true. The mean time to extinction is actually minimized at an intermediate selection coefficient. A simple heuristic argument suggests why this should be true. Neutral mutations have no influence on the probability of extinction, whereas lethal mutations are eliminated in a single generation and have no cumulative effect. However, mutations with mildly deleterious effects can be maintained for long periods of time, and as soon as each individual carries at least one (not necessarily the same) mutation, a permanent reduction in mean absolute fitness occurs. We will show that the magnitude of the mutational effect that causes the highest risk of extinction can be quite small, a result that leads to the suggestion that selection might sometimes favor the spread of asexual lineages in which the individual effects of mutations are most damaging.

Because of the highly stochastic nature of the mutational meltdown process, most of our previous work on the phenomenon has relied on computer simulation. To provide a deeper heuristic understanding of the process, the extensive computer simulations presented in this paper are accompanied by some simple numerical approximations, which provide quite reasonable results for a range of situations.

Finally, we give some consideration to the implications of Muller's ratchet for the survival of sexual populations. Although recombination can retard the accumulation of deleterious mutations in the nuclear genome of sexual species, it is an open question as to whether small sexual populations suffer from mutational meltdowns in the genomes of their organelles. From the standpoint of the mitochondrial genome (and chloroplasts in plants), sexual organisms are simply carriers of small asexual lineages, which are absolutely vital to survival. Because mitochondrial DNA (mtDNA) is usually inherited clonally through one parent, it should be subject to Muller's ratchet.

\section{COMPUTER Simulations}

We studied the influence of viability mutations on the mean extinction time for populations growing in discrete generations with two types of population-density regulation. In both models, viability selection on juveniles follows density dependence within each generation.

(1) Under the density-dependent model, investigated earlier without mutation by Gabriel and Bürger (1992), the expected population size at the beginning of generation $t+1$ is

$$
N^{\prime}(t+1)=\frac{N(t) e^{r}}{1+N(t)\left(e^{r}-1\right) / K},
$$

where $e^{r}$ is the maximum growth rate, $K$ is the carrying capacity, and $N(t)$ is the number of reproducing adults. This discrete version of the logistic model does not exhibit chaotic or cyclic behavior (May 1981). Under the assumption of independent, Poisson-distributed family sizes, the actual population size at the beginning of generation $t+1, N_{0}(t+1)$, was drawn from a Poisson distribution with expectation $N^{\prime}(t+1)$ for that generation. With this model, mutation accumulation leads to a decline in the expected number of offspring over time, and there is always a probability of extinction caused by demographic stochasticity (the chance absence of offspring production is equal to $\left.e^{-N^{2}(t)}\right)$.

(2) In the high-fecundity model, offspring recruitment is assumed to be substantial enough to bring the population back up to the carrying capacity at the beginning of each generation, and there is no extinction caused by demographic stochasticity. Such conditions put an obvious upper limit on the time to extinction for any set of genetic parameters.

Starting with mutation-free genotypes, individuals incurred new mutations each generation following a Poisson distribution with expectation $\mu$. The probability of survival to maturity for an individual with $n$ mutations was determined by the fitness function $W_{n}=(1-s)^{n}$, where $s$ is the fractional reduction in viability caused by a single mutation. The $N(t+1)$ reproductive adults in generation $t+1$ were identified by drawing uniformly distributed random numbers in the interval 0 to 1 for each of the $N_{0}(t+1)$ juveniles and imposing mortality whenever the random number was greater than $W_{n}$. Extinction occurs whenever all $N_{0}$ offspring die.

As a check on the simulations, the mean times 
to extinction for the special cases in which $s=$ 0 and $s=1$ were calculated numerically using Markov-chain theory. For the density-dependent model, the case $s=0$ has been described elsewhere (Gabriel and Bürger 1992), and the case $s=1$ is essentially the same except that $r$ and $K$ must be replaced with $r_{1}=r-\mu$ and $K_{1}$ $=K\left[e^{(r-\mu)}-1\right] /\left(e^{r}-1\right)$ (Appendix A). For large $r$ (e.g., $r \geq 2$ ), the mean time to extinction $\left(t_{E}\right)$ is approximately $e^{K}$ if $s=0$ (Gabriel and Bürger 1992 ), and $e^{K_{I}}$ if $s=1$. For the high-fecundity model, there is no extinction when $s=0$, and $t_{E}$ $=\left[1-e^{-\mu}\right]^{-K}$ when $s=1$.

\section{Numerical Approximations}

I. Quantitative-Genetic Approach. - For situations in which the number of mutations arising in the population per generation is reasonably high, a fair amount of variation is expected for the number of mutations $n$ carried by different individuals. Under such circumstances, as a first approximation, $n$ can be treated as a continuously distributed quantitative character with mean $\bar{n}$ and variance $\sigma_{n}^{2}$ (see Appendix B for details).

Because mutations are assumed to arise in a Poisson fashion, they increase the mean and variance by $\mu$ per generation, thus prior to selection

$$
\begin{aligned}
\bar{n}_{(\mu)}(t) & =\bar{n}(t)+\mu, \\
\sigma_{n(\mu)}^{2}(t) & =\sigma_{n}^{2}(t)+\mu .
\end{aligned}
$$

The subsequent reduction in $\bar{n}$ by selection is obtained by combining equations (2a) and (B8),

$$
\begin{aligned}
\bar{n}(t+1) & \simeq \bar{n}_{(\mu)}(t)-s \sigma_{n(\mu)}^{2}(t) \\
& =\bar{n}(t)+\mu(1-s)-s \sigma_{n}^{2}(t),
\end{aligned}
$$

assuming selection is weak. The main difficulty with this expression is the need for an expression for $\sigma_{n}^{2}(t)$.

To a large extent, the dynamics of genetic variance in finite populations are controlled by the input caused by new mutations and the loss caused by sampling, even when the mutations are under selection (Bürger et al. 1989). Sampling causes an expected reduction in the variance in two ways. First, of the $N^{\prime}(t)$ individuals expected at the beginning of a generation, only an expected $N(t) \simeq N^{\prime}(t) \bar{W}(t)$ survive. This can be shown to reduce the variance to a fraction $N^{\prime}(t)[N(t)-1] /$ $\left[N^{\prime}(t)-1\right] N(t)$ of that prior to mortality, under the assumption that the variance in $n$ is low rel- ative to the mean. Second, sampling of the surviving adults for progeny production leads to a further reduction of the variance by the fraction $1 / N^{\prime}(t+1)$. The most successful approximation that we could find for the influence of selection on the variance of $n$ is to simply reduce the variation further by the fraction $(1-s)$. Such a reduction is equivalent to the expectation when the number of mutations in the population in excess of the minimum number is a Poissondistributed variable among individuals. Thus, as a first approximation, we allow the dynamics of the genetic variance to be defined by

$$
\sigma_{n}^{2}(t+1) \simeq(1-s) \gamma(t)\left[\sigma_{n}^{2}(t)+\mu\right],
$$

where $\gamma(t)=\lambda\left[N^{\prime}(t+1)\right] \lambda[N(t)] / \lambda\left[N^{\prime}(t)\right]$, with $\lambda[x]$ being the function $[1-(1 / x)]$, and $N^{\prime}(t)$ being generated by equation (1). Similar approaches were employed by Pamilo et al. (1987) and Charlesworth (1990) to predict the accumulation of mutations, but they assumed a constant number of reproductive adults (an infinite number in the latter study).

Iteration of equations (1), (3), and (4) can be used to obtain an approximate probability distribution of extinction times. Extinction in any generation requires that all $N^{\prime}(t)$ individuals die or that the survivors produce no offspring by chance. Therefore, the conditional probability of population survival from generation $t$ to $t+1$ is approximately

$$
P(t)=\left\{1-[d(t)]^{N^{(}(t)}\right\}\left[1-e^{-N^{\prime}(t+1)}\right],
$$

where we let $d(t)=1-(1-s)^{\bar{n}(t)}$ approximate the probability of individual mortality in generation $t$ [see equation (B10)], and $e^{-N^{\prime}(t+1)}$ is the Poisson probability of no offspring production when the expected number is $N^{\prime}(t+1)$. The probability distribution for extinction times is

$$
p_{E}(t)=[1-P(t)] \prod_{t=0}^{t-1} P(i) .
$$

When the mutation rate is moderately high and selection is weak relative to drift $(s \leq 1 / K)$, this approach yields results that are very similar to the mean extinction times obtained from simulations of the high-fecundity model (table 1). Although they are not shown, for the same range of parameter values, the standard deviations of $t_{E}$ obtained with the quantitative-genetic approximation are also very similar to the simulation results. Table 1 also shows that with low 
$s$, the mean extinction time depends only weakly on $K$ for large $K$, as pointed out earlier in Lynch and Gabriel (1990).

In the case of the density-dependent model, the numerical procedure tends to overestimate $t_{E}$. However, for large $K\left(>10^{2}\right)$ and weak selection $(s<0.01)$, the quantitative-genetic approach yields results that are in rough accord with those obtained by simulation.

II. Fixation-Probability Approach. - When the mutation rate is fairly high, the minimum number of mutations per individual is not necessarily the same as the number of mutations fixed in the population. But provided the number of mutations entering the population each generation is sufficiently low $(\mu K \ll 1)$, then any new mutation will either be lost or go to fixation prior to the appearance of the next mutation. Under these circumstances, a population essentially never contains more than two types of individuals, and the rate at which the ratchet clicks is equivalent to the fixation rate. For the high-fecundity model, the probability of fixation for new mutant alleles is simply a function of $s$ and $K$. It remains constant throughout the entire period of mutation accumulation and can be computed with a transition-matrix approach (Appendix C).

Knowing the fixation probability of a mutant gene, it is possible to approximate the mean extinction time in the following way. Each generation, an expected $\mu K$ mutations arise, each of which has a probability of fixation of $u_{F}$. Thus, the expected time between fixation events is $t_{M}$ $=1 /\left(\mu K u_{F}\right)$. Given that a fixation occurs, the time from appearance to fixation is expected to be small relative to the time between fixation events (Appendix C), thus the probability of extinction each generation in the period between $n$th and $(n+1)$ th fixation is approximately $\theta(n)=[1-$ $\left.(1-s)^{n}\right]^{K}$. Letting $L(n)=\prod_{i=0}^{n}[1-\theta(i)]^{\tau_{M}}$ be the approximate probability that a population still survives prior to the fixation of the $n$th mutation, the mean time to extinction is estimated by

$$
\bar{t}_{E}=\sum_{n=0}^{\infty} L(n)\left[\bar{t}_{M}+p_{E}(n+1) \bar{t}_{E}(n+1)\right],
$$

where $p_{\mathrm{E}}(n+1)=1-[1-\theta(n+1)]^{\tau_{M}}$ is the probability of extinction during the interval between the fixation of the $(n+1)$ th and $(n+2)$ th mutations, and $t_{E}(n+1)=\Sigma_{t=1}^{i_{M}}[1-\theta(n+1)]^{\prime} \theta(n$ $+1) i / p_{E}(n+1)$ is the mean time to extinction during that interval, conditional on it occurring.

Table 2 compares values of $t_{E}$ obtained by simulation with those estimated by the fixation-
TABLE 1. Comparison of mean extinction times obtained by computer simulation (OBS) with those estimated by the quantitative-genetic approximation (QG). In all cases, the zygotic mutation rate is $\mu=0.5$. For each set of parameter values, 1024 simulations were performed.

\begin{tabular}{|c|c|c|c|c|}
\hline$K$ & $r$ & $s$ & OBS & QG \\
\hline \multicolumn{5}{|c|}{ High-fecundity model } \\
\hline \multirow[t]{4}{*}{8} & & 0.1 & 38 & 38 \\
\hline & & 0.01 & 197 & 200 \\
\hline & & 0.001 & 1342 & 1351 \\
\hline & & 0.0001 & 9723 & 9725 \\
\hline \multirow[t]{4}{*}{32} & & 0.1 & 96 & 100 \\
\hline & & 0.01 & 472 & 475 \\
\hline & & 0.001 & 3578 & 3573 \\
\hline & & 0.0001 & 30,225 & 30,091 \\
\hline \multirow[t]{4}{*}{128} & & 0.1 & 206 & 263 \\
\hline & & 0.01 & 846 & 899 \\
\hline & & 0.001 & 6409 & 6365 \\
\hline & & 0.0001 & 56,210 & 55,918 \\
\hline \multirow[t]{4}{*}{512} & & 0.1 & 428 & 806 \\
\hline & & 0.01 & 1308 & 1726 \\
\hline & & 0.001 & 9529 & 9785 \\
\hline & & 0.0001 & 84,131 & 83,860 \\
\hline \multicolumn{5}{|c|}{ Density-dependent model } \\
\hline \multirow[t]{6}{*}{8} & 0.5 & 0.1 & 12 & 14 \\
\hline & & 0.01 & 32 & 71 \\
\hline & & 0.001 & 65 & 385 \\
\hline & 5.0 & 0.1 & 31 & 38 \\
\hline & & 0.01 & 137 & 192 \\
\hline & & 0.001 & 574 & 1073 \\
\hline \multirow[t]{6}{*}{32} & 0.5 & 0.1 & 23 & 26 \\
\hline & & 0.01 & 91 & 120 \\
\hline & & 0.001 & 521 & 916 \\
\hline & 5.0 & 0.1 & 91 & 98 \\
\hline & & 0.01 & 441 & 467 \\
\hline & & 0.001 & 3279 & 3522 \\
\hline \multirow[t]{6}{*}{128} & 0.5 & 0.1 & 35 & 38 \\
\hline & & 0.01 & 137 & 157 \\
\hline & & 0.001 & 873 & 1075 \\
\hline & 5.0 & 0.1 & 195 & 252 \\
\hline & & 0.01 & 793 & 862 \\
\hline & & 0.001 & 6032 & 6138 \\
\hline \multirow[t]{6}{*}{512} & 0.5 & 0.1 & 49 & 56 \\
\hline & & 0.01 & 175 & 206 \\
\hline & & 0.001 & 1095 & 1240 \\
\hline & 5.0 & 0.1 & 394 & 762 \\
\hline & & 0.01 & 1149 & 1591 \\
\hline & & 0.001 & 8370 & 8949 \\
\hline
\end{tabular}

probability approach for various combinations of $K$ and $\mu$ satisfying $K \mu<0.5$. The numerical approximation tends to overestimate the simulation results. However, when selection is weak $(2 K s<1)$, the fixation-probability approximation is in good accord with the simulations.

III. Quasi-Steady-State Approach. - For very large $K$ (at least on the order of billions) and weak mutation pressure $(\mu \ll 1)$, a simpler determin- 
TABLE 2. Comparison of mean extinction times obtained by computer simulation (OBS) with those estimated by the fixation-probability (FP) approximation. All simulations are with the high-fecundity model. For each set of parameter values, 1024 simulations were performed.

\begin{tabular}{|c|c|c|c|c|}
\hline$K$ & $\mu$ & $s$ & OBS & FP \\
\hline \multirow[t]{12}{*}{2} & 0.1 & 0.46 & 26 & 32 \\
\hline & & 0.28 & 25 & 28 \\
\hline & & 0.10 & 35 & 39 \\
\hline & & 0.01 & 139 & 140 \\
\hline & 0.01 & 0.46 & 268 & 275 \\
\hline & & 0.28 & 176 & 191 \\
\hline & & 0.10 & 184 & 201 \\
\hline & & 0.01 & 643 & 668 \\
\hline & 0.001 & 0.46 & 2820 & 2709 \\
\hline & & 0.28 & 1836 & 1791 \\
\hline & & 0.10 & 1375 & 1322 \\
\hline & & 0.01 & 3092 & 3299 \\
\hline \multirow[t]{8}{*}{8} & 0.01 & 0.46 & 20,804 & 25,905 \\
\hline & & 0.28 & 2598 & 4340 \\
\hline & & 0.10 & 1295 & 1663 \\
\hline & & 0.01 & 5609 & 5764 \\
\hline & 0.001 & 0.46 & 240,888 & 254,564 \\
\hline & & 0.28 & 25,453 & 34,916 \\
\hline & & 0.10 & 10,189 & 12,314 \\
\hline & & 0.01 & 41,681 & 42,488 \\
\hline \multirow[t]{4}{*}{32} & 0.01 & 0.10 & 16,917 & 129,073 \\
\hline & & 0.01 & 18,492 & 21,517 \\
\hline & 0.001 & 0.10 & 246,183 & $1,134,930$ \\
\hline & & 0.01 & 161,154 & 186,426 \\
\hline
\end{tabular}

istic approximation can be obtained for the highfecundity model. Assuming an initial "steadystate" Poisson distribution of mutations per individual, as suggested by earlier selection-mutation balance theory (Haigh 1978), the population size prior to reproduction is reduced to $K e^{-\mu}$ by selection (see Appendix D). A further decline in the expected population size occurs in the following generations as Muller's ratchet operates, with each incremental loss of the least loaded mutation class reducing mean viability by the factor $(1-s)$. In any generation, the fraction of the population in the least loaded mutation class is approximately $e^{-\mu(1-s) / s}$ (see Appendix $\mathrm{D}$ ). Therefore, the probability that none of the $K$ progeny are drawn from this class (i.e., that the least loaded class is lost) is approximately $\phi=\left[1-e^{-\mu(1-s) / s}\right]^{K}$ in any generation. If restoration of the steady-state Poisson distribution of mutation classes occurs rapidly compared with the rate of loss of least-loaded classes (i.e., if $K$ is sufficiently large and/or $\mu / s$ is not too large), then the expected census population size in generation $t$ can be approximated by

$$
N(t) \simeq K e^{-\mu}(1-s)^{\phi t} .
$$

The approximate expected time to extinction is then taken to be the generation at which $N(t)$ falls just below 1

$$
\begin{gathered}
\bar{t}_{E} \simeq-\frac{\ln \left(K e^{-\mu}\right)}{\phi \cdot \ln (1-s)} \simeq \frac{\ln K-\mu}{\phi s} . \\
\text { THE INFLUENCE OF } s \text { ON } \\
\text { EXTINCTION TIME }
\end{gathered}
$$

It has long been known that the equilibrium mean fitness of an infinite population subject to recurrent deleterious mutation is approximately independent of the severity of the mutations (Haldane 1937). However, for finite populations, the magnitude of $s$ has a substantial influence on the time to extinction. Our simulations, for various combinations of $r$ and $K$, show that there is a minimum time to extinction at an intermediate value of $s$, which we refer to as $s^{*}$ below. This critical value declines with increasing $r$ and $K$ (figs. 1, 2), and it was obscured in our earlier work (Lynch and Gabriel 1990) where we confined attention to $s<0.5$ and small $K$. The mean time to extinction at $s^{*}$ can be several orders of magnitude less than when the individual effects of mutations are more or less deleterious.

For the special case in which both $r$ and $K$ are large, $s^{*}$ can be approximated by use of equation (9). Solving by differentiation for the value of $s$ that minimizes $t$,

$$
\frac{K\left[\mu\left(1-s^{*}\right) / s^{*}\right]}{e^{\mu\left(1-s^{*}\right) / s^{*}}-1}=-\frac{s^{*}}{\ln \left(1-s^{*}\right)},
$$

which can be solved numerically. Figure 2 shows that this approach yields estimates of $s^{*}$ that are quite similar to those obtained from simulations at large $K$. Note that $s^{*}$ increases with the genomic mutation rate, but provided the latter does not exceed $1, s^{*}$ is expected to be on the order of 0.1 or less when $K$ is more than a few hundred individuals. The nearly linear increase of $s^{*}$ with $\mu$ can be understood from equation (10) which, assuming $\ln \left(1-s^{*}\right) \simeq-s^{*}$, reduces to $s^{*}=\mu /(x$ $+\mu) \simeq \mu / x$ with $x$ being the solution of $K x=e^{x}$ -1 .

\section{An Evolutionary Advantage for DELETERIOUS Mutation}

The fact that the time to extinction is minimized at some intermediate value of $s$ has an interesting implication for the evolution of repair systems. Most DNA damages, such as double- 


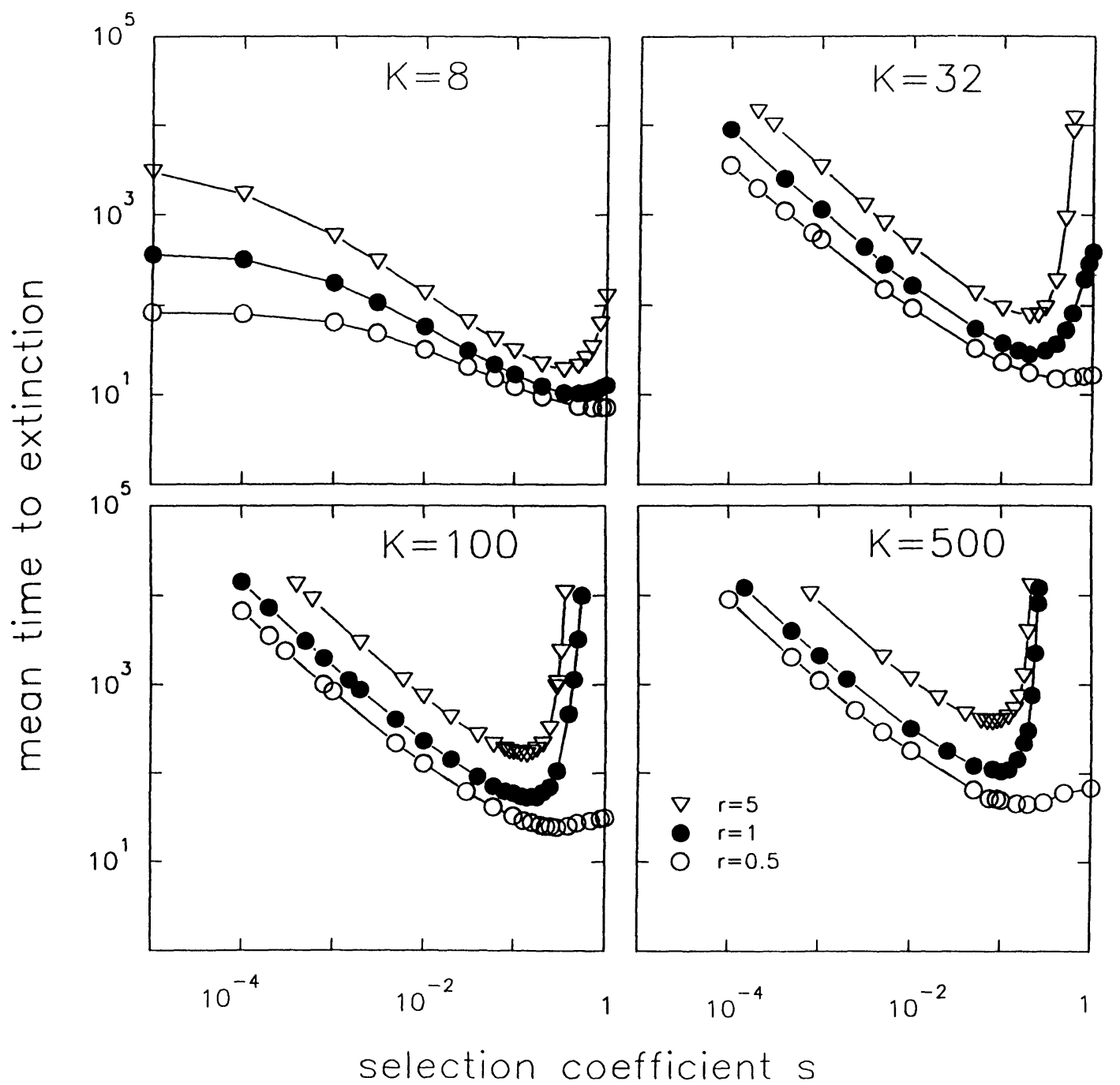

Fig. 1. Mean time to extinction for asexual populations under density-dependent growth carrying capacity $K$ and maximim growth rate $r$. A genomic mutation rate of $\mu=0.5$ is assumed.

strand breaks, are lethal $(s=1)$ if left unrepaired, in which case they are eliminated efficiently from the population. However, unless the repair system is perfect, the mechanisms of repair can induce secondary mutational changes (base-pair substitutions, insertions, or deletions) for which $0 \geq s \geq 1$. Similarly, by imperfect proofreading, mechanisms may reduce the effects of a mutation to something less deleterious, although not necessarily $s=0$. In either case, by reducing the efficiency of selection, an imperfect repair system can facilitate the accumulation of deleterious mutations in an asexual lineage.

Consider mutations (or DNA damage) which, if unrepaired, have effects in excess of $s^{*}$. Partial repair of such mutations will improve the fitness of the individual carriers, but unless the reduction in $s$ exceeds a threshold value, it will actually enhance the likelihood of lineage extinction. That is, for mutations with large effects, it is not until a critical level of repair efficiency is exceeded that repair becomes advantageous at the population level. Thus, if $s^{*}$ is very low, as it appears to be for populations with more than 100 individuals or so, it might be advantageous for asexual populations to simply accept deleterious mutations and DNA damage without repairing them unless repair is nearly perfect. Although this would cause more selective deaths each generation, it would also reduce the long-term cumulative damage 


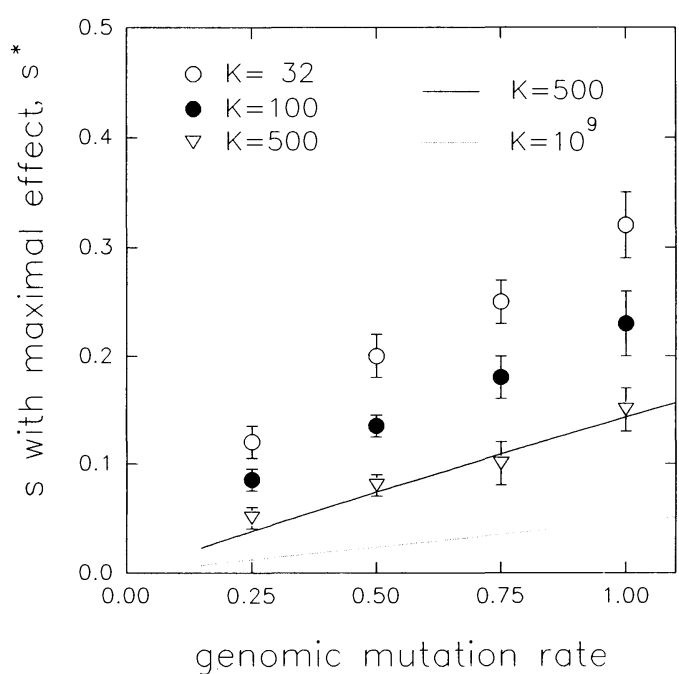

Fig. 2. The selection coefficient $s^{*}$ that causes the maximum vulnerability to extinction as a function of the carrying capacity $K$ and the genomic mutation rate $\mu$. Results are given for the high-fecundity model. The estimates and their standard errors were obtained through simulations, and the lines by the analytical approximation, equation (10), described in the text

resulting from the accumulation of more mildly deleterious mutations.

These arguments suggest that asexual lineages in which individuals are highly sensitive to deleterious mutations may be advanced by selection. To verify this, we considered a simple metapopulation structure-two demes interconnected by migration. Initially, each deme was fixed with a clonal type subject to a unique $s$, but mutation rates were identical for individuals of both types. The mutations incurred by each individual had the same $s$ as their ancestral form. Both demes were assumed to have sufficiently high $r$ that they were returned to $K$ offspring at the beginning of each surviving generation, and both demes had identical $K$. The expected number of immigrants to each deme was $m K$ per generation, with the actual number being obtained from a Poisson distribution, these being drawn randomly from the surviving individuals in the opposite deme. Whenever a deme went extinct, it was immediately colonized by $K$ offspring from the surviving deme, and each stochastic run proceeded until the entire metapopulation had become fixed for one of the $s$-types or gone extinct.

Figure 3 illustrates some results for the case in which $m=0.1$ and $\mu=0.6$. Simulations were done for a range of $s_{1}$ in the first deme, keeping

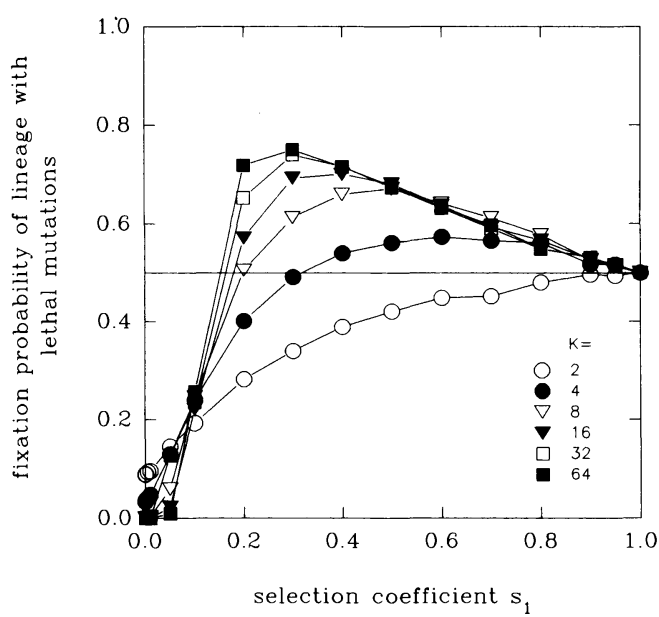

Fig. 3. The probability of fixation of an asexual lineage in which all mutations are lethal $\left(s_{2}=1\right)$ when starting at equal frequency with a lineage in which $s_{1}$ $\leq 1$. A metapopulation structure involving two demes with migration rate 0.1 , and described in more detail in the text, is assumed. Results are given for several different carrying capacities for the local demes.

$s_{2}=1$ (lethal mutations) constant in the second. When $s_{1}$ is sufficiently low, the probability of fixation of the $s_{1}$ type exceeds that of the $s_{2}$ type. However, as $s_{1}$ increases, the probability of fixation of the $s_{2}$ type increases and eventually exceeds 0.5 . Moreover, the range of $s_{1}$ over which the $s_{2}$ type has an advantage expands with increasing $K$, as expected from the patterns in figure 1. Although these results are not an exhaustive coverage of the parameter space, they are sufficient to show that a lineage for which all mutations are lethal can have a high probability of spreading to fixation in a metapopulation containing individuals with lower individual sensitivity to mutations.

\section{Mitochondrial Mutational Meltdowns}

As noted above, the relevance of Muller's ratchet extends beyond asexual species. Almost all higher organisms contain cytoplasmic genomes in mitochondria (and in plants, in chloroplasts). In most cases, cytoplasmic inheritance is uniparental without recombination (Birky 1978). In principle, such vertical transmission of isolated populations of organelles should be highly conducive to the operation of Muller's ratchet, even in sexual species, because the "effective number of organellar genomes" passing through individual germlines is usually fewer than a few hundred and in some cases on the order of three 
or four (Rand and Harrison 1986; Solignac et al. 1987; Ashley et al. 1989; Koehler et al. 1991). As the evidence is now fairly compelling that mutation accumulation leads to the extinction of small asexual populations, the question arises as to whether mitochondrial lineages suffer the same fate (Hastings 1992).

It seems likely that there is a substantial difference between the genomic mutation rates of nuclei and mitochondria. The genome size of a typical animal mitochondrion is on the order of $10^{-5}$ to $10^{-3}$ of that of its associated nuclear genome. However, in most cases, especially in animals, the mitochondrial genome consists almost entirely of coding regions for genes of vital importance, whereas a large fraction of the nuclear genome is nontranscribed and/or has no known function. Thus, one might expect the incidence of nonneutral mutations in the mitochondrion relative to the nucleus to be somewhat greater than their relative genome sizes. Some data have been cited as evidence that the mutation rate per base pair in the mitochondrion is elevated in comparison with that in the nuclear genome (Brown et al. 1979), although it is uncertain whether that is the general case (Lynch 1993), and in any event it is unlikely to be inflated by more than a fewfold. Based on these arguments, and evidence that the rate of deleterious mutation is on the order of 0.1 to 1 per nuclear genome per generation (Kondrashov 1988), $\mu=10^{-4}$ to $10^{-1}$ would appear the likely range of the genomic deleterious mutation rate for the animal mitochondrion.

We will consider two, not necessarily independent, ways in which mutation accumulation might result in extinction of a mitochondrial lineage. First, mutations that cause a reduction in replication or survival of their bearers are subject to selection within the host cell (Backer and Birky 1985; Gingold 1988). A significant base substitution or insertion in an origin of replication is an example of such a mutation. Under this scenario, the mutational-meltdown process can be modeled as described above. The "population" consists of a vertically transmitted group of mitochondria, with each mitochondrion acquiring an expected $\mu$ mutations per host generation with a reduction of replicability or viability per mutation equal to $s$. If we assume that most such mutations are confined to the origin of replication, or at least to a small portion of the mitochondrial genome, then $K \mu$ will almost certainly be much less than one. Thus, if there is a cellular mechanism for regulating the number of mitochondria in gametes at $K$, which seems to be the case (Boffey and Lloyd 1988), and if the individual effects of mutations are also sufficiently small $(2 K s<1)$, the fixation-probability approach will provide accurate estimates of the mean time to extinction of an individual mitochondrial lineage caused by accumulation of mutations with deleterious effects on replication. This approach, combined with direct simulations where $2 K s>1$, was used to generate the results in figure 4A. Even for very small $K$ and the most deleterious values of $s$, the mean extinction time for a mitochondrial lineage via this mechanism is on the order of thousands to millions of generations (host-cell divisions). Obviously, it cannot be less than $1 / \mu$, the expected time to appearance of the first mutation.

Second, it is conceivable that many of the mutations arising in mitochondria cause a reduction in the fitness of the host cell without causing any change in the relative viability or replicability of the mitochondria themselves. Such a situation seems possible because essentially all of the genes sequestered in the mitochondrion are either involved in the metabolism of the host cell or in the translation of those genes and are not used directly in constructing new mitochondria. For example, a mutational change in a protein locus may have an important influence on the physiology of the host with no consequences for the relative replicative ability of the mutant mitochondrial DNA (mtDNA) molecule. In this case, selection operates at the level of the host, with changes in mitochondrial types within a host individual being completely neutral with respect to each other. To model this process, we again rely on the fixation-probability approach. In this case, however, the fixation probability $\left(u_{\mathrm{F}}\right)$ of a new mutation is simply its initial frequency, thus the expected time between fixation events is $\bar{t}_{M}$ $=1 / \mu$. Each fixed mutation is assumed to reduce the viability of the host by $s$, thus the probability that the host dies (and the mitochondrial lineage goes extinct) in each generation in the period between the $n$th and $(n+1)$ th fixations is approximately $\theta(n)=1-(1-s)^{n}$. The computation of $\bar{t}_{E}$ then follows from equation (7).

It seems very difficult, maybe impossible, for mitochondrial lineages to avoid this kind of meltdown, because selection does not operate within the lineage. With genomic mutation rates on the order of $10^{-2}$ to $10^{-3}$, extinction of mitochondrial lineages is expected in a few hundred 

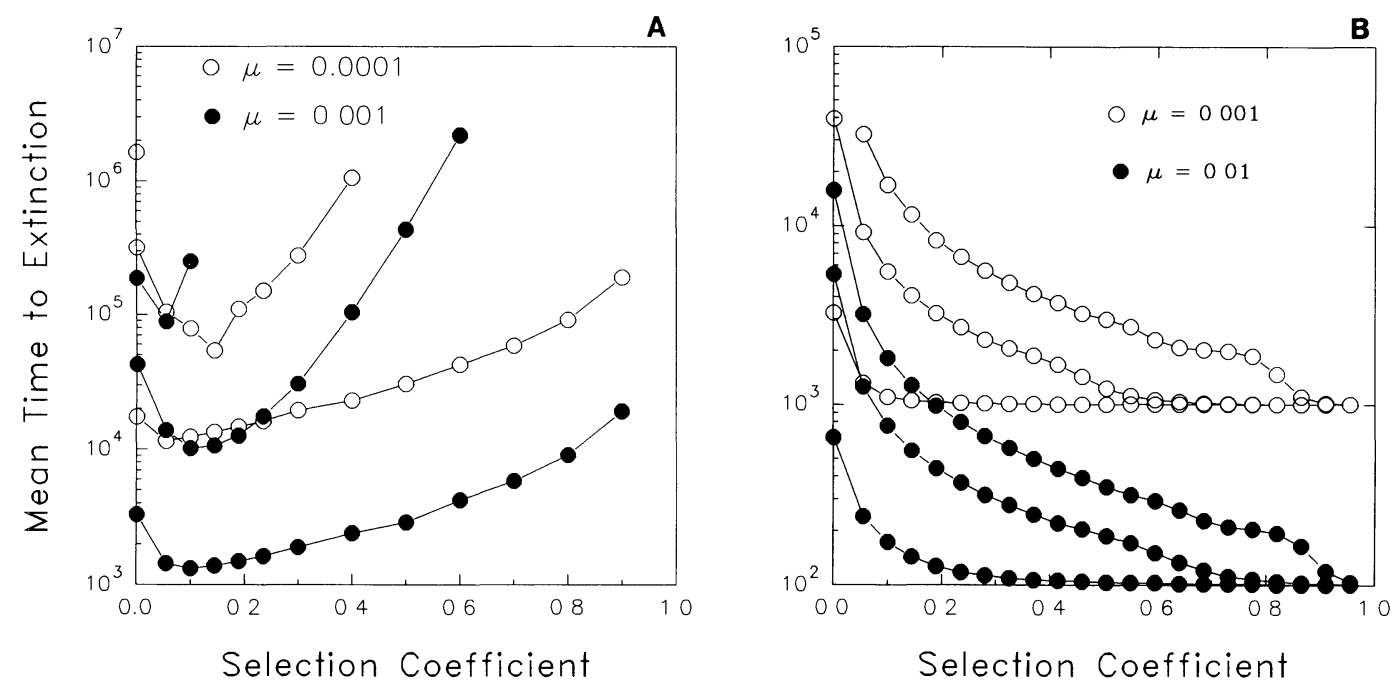

FIG. 4. Mean times to extinction for mitochondrial genomes under two situations. Left, the mutations influence the replicability of their mitochondrial bearers. Right, the mutations affect the fixation of the host cell but have no influence on the mitochondrion's ability to replicate. In both cases, the results are given for the "highfecundity" model-hosts inherit exactly $K$ organelles each generation. For a given mutation rate, the three curves in ascending order are for $K=2,8$, and 32. Note that the left-most points are for $s=0.01$.

to several thousand host-cell generations, and the mean extinction time asymptotically approaches $1 / \mu$ at high $s$ (fig. 4B). Selection among hosts (and possibly cells within hosts) can, of course, sort out more from less mutated lineages, thus these extinction times refer only to individual lines of descent, not to the entire population of hosts. Nevertheless, the observed results should be viewed as liberal upper bounds on the mean lineage extinction time because it was assumed that (1) viability is initially equal to one, (2) the lineage of mitochondria is regulated to exactly $K$ individuals per host in each surviving generation, and (3) there are no other sources of host mortality.

\section{Discussion}

Although it has long been known that mildly deleterious mutations can accumulate in small populations subject to random genetic drift, little attention has been given to the consequences of the loss in individual fitness. Our objective has been to ascertain the general qualitative features of the role of deleterious mutation in population extinction. Our earlier work on the mutational meltdown (Lynch and Gabriel 1990; Gabriel et al. 1991) relied largely on computer simulation, which depending upon the parameter values, can be very time consuming and not necessarily very revealing of the mechanisms leading to extinc- tion. Thus, our success at finding some analytical approximations should be of some value in future research. From this perspective, our major findings are the following. (1) If most individuals incur new mildly deleterious mutations every couple of generations or so, and $K$ is smaller than a few hundred, the mutational-meltdown process can be approximated very well by treating $n$ like a continuously distributed quantitative trait. (2) However, if the total number of mutations arising in a population per generation is less than one, and selection is weak, the process can be approximated closely by a fixation-probability approach. (3) When the population size is very large, and the mutation rate is low, the process can be modeled by assuming that the form of the distribution of mutation number remains close to the Poisson expected in an infinite population but with the mean gradually shifting to the right via random genetic drift.

The results of this study confirm and extend our previous conclusion that the mean time to extinction for an obligately asexual lineage of moderate size is unlikely to exceed a few thousand generations. For example, for the high-fecundity model that we utilized, there is no environmental source of mortality, no deleterious genes in the base population, no demographic stochasticity, and no density dependence. Yet even in such an extremely benign situation, the 
expected extinction time for a population with a carrying capacity of 512 individuals is approximately 1300 generations with $\mu=0.5$ and $s=$ 0.01 (table 1). Because it is likely that the true value of $\mu$ is somewhat higher than 0.5 (Kondrashov 1988), and most of the factors just mentioned will usually be present, the time to extinction for any natural population will almost certainly be at least an order of magnitude below most of our reported results.

The models that we have employed for density-dependent regulation are, of course, somewhat arbitrary. Our attention has been confined to the situation in which the environment is characterized by a carrying capacity $K$, which defines the number of newborns that can be supported, and viability selection follows offspring production. Under these conditions, the number of reproductive adults declines progressively below $K$ as the mutation load increases. Elsewhere (Lynch et al. 1993), we have considered the case in which the carrying capacity defines the number of adults that the environment can support. In that model, each surviving adult produces an expected $e^{r}$ offspring, and the newborn cohort is brought down to the carrying capacity by selection and, if necessary, random culling. Hence, the number of reproductive adults remains at $K$ until mean viability drops below $e^{-r}$. The mean time to extinction is enhanced when density dependence is enforced at the adult stage, but not greatly so, and none of the conclusions of this paper are altered qualitatively.

By definition, unconditionally deleterious mutations are disadvantageous to the individuals carrying them. Thus, it is reasonable to expect that throughout evolutionary history there has been a strong selective advantage at the individual level for reduced sensitivity to DNA sequence changes and damage. Yet, whereas evolution has given rise to a variety of intricate cellular and developmental mechanisms by which organisms can repair damage to their phenotypes, DNA damage is often permanent. Our results suggest that if the average effect of mutations is to reduce individual fitness by more than $1 \%$ or so, selection in large asexual populations may lead to the dominance of lineages with poor mechanisms for repairing certain kinds of DNA damage and/or mutations. Ultimately, the selective advantage of any repair mechanism will depend on both the reduction of the mutation rate (through perfect repair) and on the change in the distribution of $s$ (for imperfectly repaired mu- tations). We confined our attention to the latter issue, showing only the feasibility of an evolution trajectory towards reduced repair and highlighting some of the criteria that are necessary for the opposite to occur.

Our results may help explain why most of the evolutionarily old lineages of asexual organisms (bacteria, blue-green algae, and some fungi) are haploid. Little is known about the relative repair capabilities of different types of organisms. However, double-strand breaks, a major source of DNA damage, are impossible to repair in a haploid cell if they occur prior to DNA replication, and gene conversion cannot be accomplished either. Thus, haploidy forces asexual individuals to accept many types of mutations and DNA damage that can be corrected at least partially in a diploid setting. It might be argued that haploidy is favored at the individual level because it allows more rapid rates of cell division, but data from bacteria suggest that cell division rates are limited by protein- rather than DNA-synthesis ( F. Stahl, pers. comm. 1992). It has been suggested that diploidy is advantageous in sexual species because it allows the effects of deleterious mutations to be masked at least partially (Kondrashov and Crow 1991; Perrot et al. 1991). However, because mutations are expected to accumulate more rapidly in asexual than in sexual species, this hypothesis does not seem to explain the rarity of diploidy in long-lived asexual lineages.

Prokaryotes do have mechanisms for repairing single-strand damage (Szostak et al. 1983) and may do so with great accuracy. But it is interesting that mitochondria, which are believed widely to have a prokaryotic origin, have reduced their genomes to the bare minimum and have no genes at all for DNA repair. Moreover, whereas nuclear genes for the ribosomal RNAs and transfer RNAs are usually found in multiple copies, they normally are reduced to single copies in the mitochondrion. Because of this, it seems likely that mutations in the anticodon or in the amino-acid loading site of a tRNA gene will have a much more immediate effect in the mitochondrion than in a nuclear genome where redundancy can insure that a mutation in a single tRNA gene is not completely debilitating. Similar arguments can be made for the rRNA.

Why do mitochondria maximize their vulnerability to DNA-level changes? It does not seem likely that this situation arose from selection at the level of host individuals, because any level of 
mitochondrial repair would improve the host's fitness. The alternative is that the isolation of mitochondria into small asexual "demes" in some way favors the long-term proliferation of lineages with high values of $s$. Following the logic in previous paragraphs, it is clear that deleterious mutation pressure in the origin of replication could produce such a situation, because the relationship between $\bar{t}_{E}$ and $s$ is $\mathrm{U}$-shaped (fig. 4A). However, unless the mutation rate in this region of the mitochondrial genome is much higher than we assumed, this would seem to be a rather slow process. However, if most mitochondrial mutations occur outside of the origin of replication, as seems likely based on the much larger amount of DNA in the coding regions, it is unclear whether the loss of DNA-repair capacity would be selected at the level of mitochondrial lineages. Under our second model, which assumes that mutations in transcribed genes are effectively neutral within lineages, the expected time to extinction (because of host inviability) decreases with increasing $s$ (fig. 4B). If we are wrong, and such mutations are actually nonneutral, there is a possible resolution to the issue, because $t_{E}$ would be minimized at an intermediate value of $s$ as usual.

Recent studies (Holt et al. 1988; Wallace et al. 1988; Holt et al. 1990; Ballinger et al. 1992; Wallace 1992) have shown that the incidence of human mitochondrial disorders is much higher than previously expected and indicate two mechanisms whereby the extinction of mitochondrial lineages through host mortality may be accelerated well beyond the rates suggested by our simulations. First, deleterious mutations that occur outside of the origin of replication can influence the replication rate. Most notably, large deletions involving coding sequences can actually enhance the rate of replication, possibly by reducing the size of the replicating molecule. Duplications of the origin of replication have the same effect. Once they appear, such "renegade" mitochondria can lead to rapid elimination of the host lineage through metabolic impairment. Second, through their influence on the oxidative state within a mitochondrion, deleterious mutations in genes involved in oxidative phosphorylation promote a more mutagenic environment, thereby leading to an accelerated loss of host fitness. Although our understanding of the mechanisms of organelle replication and selection are still rudimentary, this discussion highlights the point that issues concerning the extinction of asexual populations via deleterious mutation are quite relevant to sexual species, even if the mutational load in the nuclear genome can be kept to a low level by recombination.

\section{ACKNOWLEDGMENTS}

We thank W. Birky, T. Hanson, A. Kondrashov, R. Lande, F. Stahl, and B. Walsh for helpful comments. Our work has been supported by a grant from Deutsche Forschungsgemeinschaft to W.G., by a grant from the Austrian Fonds zur Förderung der Wissenschaftlichen Forschung, Project P8043, to R.B. and by National Science Foundation grants BSR 8911038 and BSR 9024977, and PHS grant GM36827 to M.L. A grant from the Murdock Charitable Trust provided funds for a MasPar parallel computer upon which many of the simulations were run. We are very grateful to J. Conery and S. Jones for assistance in converting our computer programs to take advantage of that environment.

\section{Literature Cited}

Ashley, M. V., P. J. Lapis, and W. W. Hauswirth. 1989. Rapid segregation of heteroplasmic bovine mitochondria. Nucleic Acids Research 17:73257331.

Backer, J. S., and C. W. Birky, Jr. 1985. The origin of mutant cells: Mechanisms by which Saccharomyces cerevisiae produces cells homoplasmic for new mitochondrial mutants. Current Genetics 9:627-640.

Ballinger, S. W., J. M. Shoffner, E. V. Hedaya, I. Trounce, M. A. Polak, D. A. Koontz, and D. C. Wallace. 1992. Maternally transmitted diabetes and deafness associated with a $10.4 \mathrm{~kb}$ mitochondrial DNA deletion. Nature Genetics 1:11.

Bell, G. 1988a. Sex and death in the protozoa. Cambridge University Press, New York.

- 1988b. Recombination and the immortality of the germ line. Journal of Evolutionary Biology $1: 67-82$

Birky, C. W., Jr. 1978. Transmission genetics of mitochondria and chloroplasts. Annual Review of Genetics 12:471-512.

Birky, C. W., Jr., and J. B. Walsh. 1988. Effects of linkage on rates of molecular evolution. Proceedings of the National Academy of Sciences, USA 85: 6414-6418.

Boffey, S. A., and D. Lloyd, eds. 1988. The division and segregation of organelles. Cambridge University Press, New York

Brown, W. M., M. George, Jr., and A. C. Wilson. 1979. Rapid evolution of animal mitochondrial DNA Proceedings of the National Academy of Sciences, USA 76:1967-1971.

Bürger, R. 1991. Moments, cumulants, and polygenic dynamics. Journal of Mathematical Biology 30:199_ 213.

Bürger, R., G. P. Wagner, and F. Stettinger. 1989. How much heritable variation can be maintained 
in finite populations by mutation selection balance? Evolution 43:1748-1766.

Charlesworth, B. 1990. Mutation-selection balance and the evolutionary advantage of sex and recombination. Genetical Research 55:199-221.

Charlesworth, B., D. Charlesworth, and M. T. Morgan. 1990. Genetic loads and estimates of mutation rates in highly inbred plant populations. Nature 347:380 382.

Crow, J. F., and M. Kimura. 1979. Efficiency of truncation selection. Proceedings of the National Academy of Sciences, USA 76:396-399.

Crow, J. F., and M. J. Simmons. 1983. The mutation load in Drosophila. Pp. 1-35 in M. Ashburner et al., eds. The genetics and biology of Drosophila, Vol. 3c. Academic Press, New York.

Ewens, W. J. 1979. Mathematical population genetics. Springer, New York.

Felsenstein, J. 1974. The evolutionary advantage of recombination. Genetics 78:737-756.

Gabriel, W., and R. Bürger. 1992. Survival of small populations under demographic stochasiticy. Theoretical Population Biology 41:44-71.

Gabriel, W., R. Bürger, and M. Lynch. 1991. Population extinction by mutational load and emographic stochasticity. Pp. 49-59 in A. Seitz and V. Loeschke, eds. Species conservation: a populationbiological approach. Birkhäuser, Basel, Switzerland.

Gingold, E. B. 1988. The replication and segregation of yeast mitochondrial DNA. Pp. 149-170 in S. A. Boffey and D. Lloyd, eds. The division and segregation of organelles. Cambridge University Press, New York.

Haigh, J. 1978. The accumulation of deleterious genes in a population. Theoretical Population Biology 14 251-267.

Haldane, J. B. S. 1937 . The effect of variation on fitness. American Naturalist 71:337-349.

Hastings, I. M. 1992. Population genetic aspects of deleterious cytoplasmic genomes and their effect on the evolution of sexual reproduction. Genetical Research 59:215-225.

Holt, I. J., A. E. Harding, and J. A. Morgan-Hughes. 1988. Deletions of mitochondrial DNA in patients with mitochondrial myopathies. Nature 331:717719.

Holt, I. J., A. E. Harding, R. K. H. Petty, and J. A. Morgan-Hughes. 1990. A new mitochondrial disease associated with mitochondrial DNA heteroplasmy. American Journal of Human Genetics 46: 428-433.

Houle, D., D. K. Hoffmaster, S. Assimacopoulos, and B. Charlesworth. 1992. The genomic mutation rate for fitness in Drosophila. Nature 359:58-60.

Kimura, M. 1962. On the probability of fixation of mutant genes in a population. Genetics 47:713719.

Kimura, M., T. Maruyama, and J. F. Crow. 1963. The mutation load in small populations. Genetics 48:1303-1312.

Kimura, M., and T. Ohta. 1969. The average number of generations until fixation of a mutant gene in a finite population. Genetics 61:763-771.

Koehler, C. M., G. L. Lindberg, D. R. Brown, D. C. Beitz, A. E. Freeman, J. E. Mayfield, and A. M.
Myers. 1991. Replacement of bovine mitochondrial DNA by a sequence variant within one generation. Genetics 129:247-255.

Kondrashov, A. S. 1988. Deleterious mutations and the evolution of sexual reproduction. Nature 334: 435-440.

Kondrashov, A. S., and J. F. Crow. 1991. Haploidy or diploidy: Which is better? Nature 351:314-315.

Lynch, M. 1993. A method for calibrating molecular clocks and its application to animal mitochondrial DNA. Genetics 135:1197-1208.

Lynch, M., and W. Gabriel. 1990. Mutation load and the survival of small populations. Evolution 44: 1725-1737.

Lynch, M., R. Bürger, D. Butcher, and W. Gabriel. 1993. The mutational meltdown in asexual populations. Journal of Heredity 84:339-344.

May, R. M. 1981. Models for single populations. Pp. 5-29 in R. M. May, ed. Theoretical ecology. Blackwell, Oxford.

Maynard Smith, J. 1978. The evolution of sex. Cambridge University Press, Cambridge.

Melzer, A. L., and J. H. Koeslag. 1991. Mutations do not accumulate in asexual isolates capable of growth and extinction-Muller's ratchet reexamined. Evolution 45:649-655.

Muller, H. J. 1964. The relation of recombination to mutational advance. Mutation Research 1:2-9.

Pamilo, P., M. Nei, and W.-H. Li. 1987. Accumulation of mutations in sexual and asexual populations. Genetical Research 49:135-146.

Perrot, V., S. Richerd, and M. Valéro. 1991. Transition from haploidy to diploidy. Nature 351:315317.

Rand, D. M., and R. G. Harrison. 1986. Mitochondrial DNA transmission genetics in crickets. Genetics 114:955-970.

Solignac, M., J. Génermont, M. Monnerot, and J.-C. Mounolou. 1987. Drosophila mitochondrial genetics: evolution of heteroplasmy through germ line cell divisions. Genetics 117:687-696.

Szostak, J. W., T. L. Orr-Weaver, R. J. Rothstein, and F. W. Stahl. 1983. The double-strand break repair model for recombination. Cell 33:25-35.

Wallace, D. C. 1992. Mitochondrial genetics: a paradigm for aging and degenerative diseases? Science 256:628-632.

Wallace, D. C., G. Singh, M. T. Lott, J. A. Hodge, T. G. Schurr, A.M.S. Lezza, L. J. Elsas, et al. 1988. Mitochondrial DNA mutation associated with Leber's hereditary optic neuropathy. Science 242: $1427-1430$.

Corresponding Editor: B. Walsh

\section{APPENDIX A}

Assuming that all mutations are lethal (i.e., $s=1$ ), it follows that the fraction of surviving individuals is $e^{-\mu}$ because the number of mutations incurred by an individual is Poisson distributed with mean $\mu$. Using equation (1) for the expected number of offspring, a simple calculation shows that the expected number of reproductive adults is 


$$
\begin{aligned}
N(t+1) & =N^{\prime}(t+1) e^{-\mu} \\
& =e^{r_{1}} \frac{N(t)}{1+N(t)\left(e^{r_{1}}-1\right) / K_{1}},
\end{aligned}
$$

where $r_{1}=r-\mu$ and $K_{1}=K\left[e^{(r-\mu)}-1\right] /\left(e^{r}-1\right)$. Therefore, the expected population size in generation $t+1$ can be calculated formally as in a pure demographic model but with reduced $r$ and $K$ values.

\section{APPENDIX B}

We denote the $k$ th cumulant of the distribution of number of mutants per individual by $c_{k}$ (see Bürger 1991). The mean is $\bar{n}=c_{1}$ and the variance is $\sigma_{n}^{2}=c_{2}$. All cumulants of a Poisson distribution are equal to the mean. Thus, because new mutations per individual follow a Poisson distribution with mean $\mu$, and a cumulant of the sum of independent random variables is just the sum of the cumulants, that is, $c_{k}(x+y)=$ $c_{k}(x)+c_{k}(y)$, the cumulants after mutation are

$$
c_{k(\mu)}=c_{k}+\mu .
$$

If the distribution was Poisson before mutation, then it remains Poisson after mutation, and the mean is $\bar{n}$ $+\mu$.

The change caused by selection is calculated by denoting the fitness function as $W_{n}=(1-s)^{n}=e^{s^{\prime} n}$, where $s^{\prime}=\ln (1-s)$. Let $p_{n}$ denote the frequency of individuals carrying $n$ mutations before selection, and $p^{\prime}{ }_{n}$ the frequency after selection. Then,

$$
p_{n}^{\prime}=e^{s^{\prime} n} p_{n} / \bar{W},
$$

where $\bar{W}=\Sigma_{n \geq 0} e^{s^{\prime} n} p_{n}$. Multiplication of this equation by $e^{z n}$ and summation over $n$ yields

$$
\bar{W} \sum_{n \geq 0} e^{z n} p_{n}^{\prime}=\sum_{n \geq 0} e^{\left(z+s^{\prime}\right) n} p_{n}
$$

and

$$
\ln \bar{W}+\ln \sum_{n \geq 0} e^{z n} p_{n}^{\prime}=\ln \sum_{n \geq 0} e^{\left(z+s^{\prime}\right) n} p_{n} .
$$

The cumulants are the coefficients $c_{k}$ of the power series of

$$
\ln \sum_{n \geq 0} e^{z n} p_{n}=\sum_{k \geq l} \frac{z^{k}}{k !} c_{k} .
$$

Denoting the $k$ th derivative with respect to $z$ by $D^{k}$, we obtain the cumulants $c_{k}^{\prime}$ after selection by differentiation of (B4) and using (B5),

$$
\begin{aligned}
c_{k}^{\prime} & =\left.D^{k}\left(\ln \sum_{n \geq 0} e^{\left(z+s^{\prime}\right) n} p_{n}\right)\right|_{z=0} \\
& =\left.D^{k}\left(\sum_{J \geq 1} \frac{\left(z+s^{\prime}\right)^{\jmath}}{j !} c_{\jmath}\right)\right|_{z=0}
\end{aligned}
$$

For $z=0$ we obtain

$$
c_{k}^{\prime}-c_{k}=\sum_{n \geq 1} \frac{\left(s^{\prime}\right)^{n}}{n !} c_{k+n} .
$$

For weak selection, that is, $s^{\prime}=\ln (1-s) \simeq-s$ and $\left(s^{\prime}\right)^{2} \simeq 0$,

$$
c^{\prime}{ }_{k} \simeq c_{k}-s c_{k+1} .
$$

If the distribution before selection was Poisson, then it remains Poisson after selection with new mean

$$
\bar{n}^{\prime}=\bar{n}(1-s) \text {. }
$$

Equation (B5) implies that mean fitness is given by

$$
\bar{W}=\exp \sum_{n \geq 1} \frac{\left(s^{\prime}\right)^{n}}{n !} c_{n} \simeq(1-s)^{n}
$$

if $s$ is sufficiently small. For a Poisson distribution, mean fitness is exactly $\bar{W}=e^{-\tilde{n} s}$.

\section{APPENDIX C}

Consider a single mutation entering a uniform population with individual fitness equal to $W_{0}$. Prior to the appearance of the next mutation, there are $K+2$ possible states of the population - the population may be extinct (state $E$ ), or if surviving, may have state $x$, with $0 \leq x \leq K$ being the number of individuals with the mutation. Letting $p(a, b)$ be the transition probability from state $a$ to $b$, then $p(E, E)=1$ and $p(E, x)$ $=0$. The probability of extinction for a population with $x$ mutants is $p(x, E)=d_{M}^{x} d_{N}^{K-x}$, where $d_{M}=1-W_{0}(1$ $-s)$ and $d_{N}=1-W_{0}$ are the mortality probabilities of mutants and nonmutants. For populations with no mutants, $p(0, E)=d_{N}^{k}, p(0,0)=1-d_{N}^{k}$, and $p(0, x)=$ 0 for $x>0$. For populations fixed for the mutation, $p(K, E)=d_{M}^{K}, p(K, K)=1-d_{M}^{K}$, and $p(K, x)=0$ for $x \leq K$. All other elements of the transition matrix are defined by

$$
\begin{gathered}
p(x, y)=\left(\begin{array}{l}
K \\
y
\end{array}\right) \sum_{m=0}^{x}\left(\begin{array}{c}
x \\
m
\end{array}\right)\left(1-d_{M}\right)^{m} d_{M}^{x-m} \\
\cdot \sum_{n=0}^{K-x}\left(\begin{array}{c}
K-x \\
n
\end{array}\right)\left(1-d_{N}\right)^{n} d_{N}^{K-x-n} \\
\cdot \frac{m^{y} n^{K-y}}{(m+n)^{K}}
\end{gathered}
$$

which, given state $x$, accounts for all possible combinations of numbers of surviving mutants $(m)$ and nonmutants $(n)$ and the associated probability distributions of progeny populations.

Letting $\mathbf{P}$ be the transition matrix, with rows and columns in order $(E, 0,1,2, \ldots, K)$, and $\mathbf{X}(t)$ be the vector of state probabilities at time $t$, then

$$
\mathbf{X}(t)=\mathbf{P}^{t} \mathbf{X}(0)
$$

with $\mathbf{X}^{T}(0)=[0,0,1,0, \ldots, 0]$ when the population initially contains a single mutant. As $t \rightarrow \infty, \mathbf{X}^{T}(t) \rightarrow$ $\left[u_{F},\left(1-u_{F}\right), 0, \ldots, 0\right]$ provided $s>0$. That is, the mutation is lost with probability $\left(1-u_{F}\right)$ or fixed with probability $u_{F}$, in which case population extinction eventually occurs (albeit with low probability per generation when $s$ is small).

The mean time to fixation of a mutation, conditional on fixation occurring, is $t_{F}=\Sigma_{t=1}^{\infty} p_{F}(t) \cdot t / u_{F}$, where $p_{F}(t)$ $=x[K, t]-x[K,(t-1)]$, with $x[K, t]$ being the $K$ th element of $\mathbf{X}$, is the probability of fixation occurring in generation $t$. The same approach can be used to compute the mean time to loss of the mutant conditional on loss occurring $\left(t_{L}\right)$, and to compute higherorder moments for $t_{F}$ and $t_{L}$.

Results from diffusion theory are also useful in this context. Modifying Kimura's (1962) result for mutations in sexual diploid populations, we can anticipate for $K s<1$ that

$$
u_{F} \simeq \frac{1-\exp (2 s)}{1-\exp (2 K s)} .
$$


When $s \ll 1 / K$, drift becomes the dominant influence on clone frequency, and the mean time to fixation (conditional on fixation of deleterious mutations) should approximate the neutral result $(s=0)$

$$
\bar{t}_{F}=2 K(1-K) \ln \left(1-\frac{1}{K}\right)
$$

(Kimura and Ohta 1969; Ewens 1979). For large $K$, $\bar{t}_{F}$ is very close to $2 K$ under neutrality, and it is always less than this quantity when mutations are deleterious.

\section{APPENDIX D}

To study the dynamics of the mutation classes it is sufficient to consider a population wth $r=0$, because reproduction after mutation and selection does not al- ter the relative size of mutation classes. We start at $t$ $=0$ with a mutation-free population of size $N_{0}$. Let $C_{t}(t)$ be the number of individuals carrying $i$ mutations in generation $t$. In the next generation, $C_{I}(t+1)$ contains contributions from the classes 0 to $i$ according to the probability $e^{-\mu} \mu^{\prime} / j$ ! with $j$ as the number of mutations necessary to reach class $i$. Therefore, $C_{1}(t+1)$ $=\Sigma_{j=0}^{t} e^{-\mu}\left(\mu^{\prime} / j !\right) C_{t-\jmath}(t)(1-s)^{2}$. By induction, it can be shown that $C_{t}(t)=N_{0} e^{-\mu t}\left[\mu \Sigma_{j=1}^{t}(1-s)^{t} / i\right.$ !. For $r=0$, the total population size decreases as $N(t)=\Sigma_{t=0}^{\infty} C_{l}(t)$ $=N_{0} e^{(-\mu t)} e^{\{[1-(1-s) t] \mu(1-s) / s\}}$. In the limit as $t \rightarrow \infty$, which corresponds to the steady-state distribution of mutational classes, $\lim _{t \rightarrow \infty} N(t+1) / N(t)=e^{-\mu}$ and $\lim _{t \rightarrow \infty}$ $C_{l}(t) / N(t)=\left\{\mu^{2}[(1-s) / s]^{t} / i !\right\} e^{-[\mu(1-s) / s]}$. In particular, $\lim _{t \rightarrow \infty} C_{0}(t) / N(t)=e^{-\mu(1-s) / s}$. 